\title{
The multi-phase gaseous halos of star-forming late-type galaxies ${ }^{\star}$ II. Statistical analysis of key parameters
}

\author{
R. Tüllmann ${ }^{1}$, D. Breitschwerdt ${ }^{2}$, J. Rossa ${ }^{3}$, W. Pietsch ${ }^{4}$, and R.-J. Dettmar ${ }^{1}$ \\ 1 Astronomisches Institut, Ruhr-Universität Bochum, 44780 Bochum, Germany \\ e-mail: [tullmann; dettmar] @astro.rub.de \\ 2 Institut für Astronomie, Türkenschanzstrasse 17, 1180 Wien, Austria \\ e-mail: breitschwerdt@astro.univie.ac.at \\ 3 Space Telescope Science Institute, 3700 San Martin Drive, Baltimore, MD 21218, USA \\ e-mail: jrossa@stsci.edu \\ ${ }^{4}$ Max-Planck Institut für extraterrestrische Physik, Giessenbachstrasse, 85748 Garching, Germany \\ e-mail: wnp@mpe.mpg.de
}

Received 21 December 2005 / Accepted 21 April 2006

ABSTRACT

\begin{abstract}
Context. In a previous paper (Paper I) we showed that multi-phase gaseous halos of late-type spiral galaxies, detected in the radio continuum, in $\mathrm{H} \alpha$, and in X-rays, are remarkably well correlated regarding their morphology and spatial extent.

Aims. In this work we present new results from a statistical analysis to specify and quantify these phenomenological relations in more detail.

Methods. This is accomplished by investigating soft X-ray $(0.3-2.0 \mathrm{keV})$ luminosities, FIR, radio continuum $(1.4 \mathrm{GHz}), \mathrm{H} \alpha, B$-band, and UV (1550-1650 Å) luminosities for a sample of 23 edge-on late-type spiral galaxies. Typical star formation indicators, such as star formation rates (SFRs), are determined and a statistical multi-parameter/frequency correlation analysis is carried out.

Results. We find strong linear correlations, covering at least two orders of magnitude, between star formation indicators and integrated (disk+halo) luminosities in all covered wavebands. In addition to the well-established $L_{\mathrm{FIR}} / L_{1.4 \mathrm{GHz}}$ relation, we show new and highly significant linear dependencies between integrated soft X-ray luminosities and FIR, radio continuum, H $\alpha, B$-band, and UV luminosities. Moreover, integrated soft X-ray luminosities correlate well with SFRs and the energy input into the ISM by SNe. The same holds if these quantities are plotted against soft halo X-ray luminosities. Only a weak correlation exists between the dust mass of a galaxy and the corresponding X-ray luminosity. Among soft X-ray luminosities, baryonic, and H I gas masses, no significant correlations are found. There seems to exist a critical input energy by SNe into the ISM or a SFR threshold for multi-phase halos to appear. It is still not clear whether this threshold is a physical one or represents an instrument-dependent sensitivity limit.

Conclusions. These findings strongly support our previous results that multi-phase gaseous galaxy halos in late-type spiral galaxies are created and maintained by outflowing gas produced in star formation processes in the disk plane. They conflict with the concept of halos being mainly due to infalling gas from the intergalactic medium.
\end{abstract}

Key words. galaxies: formation - galaxies: halos - galaxies: ISM - galaxies: spiral - galaxies: starburst - X-rays: galaxies X-rays: ISM

\section{Introduction}

In Paper I (Tüllmann et al. 2006) we established the morphological correlation and spatial coincidence between gaseous halos in late-type spiral galaxies, traced by their radio continuum, $\mathrm{H} \alpha$, and soft diffuse $\mathrm{X}$-ray emission. We further showed that the continuum emission radiated at UV wavelengths $(\sim 210 \mathrm{~nm})$ originated in the disks of the galaxies and is well associated with diffuse ionized gas (DIG) in the halo. It is generally accepted that the non-thermal radio continuum is a reliable indicator for cosmic ray (CR) halos, that extraplanar soft X-rays are a good tracer of the hot ionized medium (HIM, e.g., produced by supernova remnants (SNRs), in superbubbles or in superwinds), and that the presence of extraplanar DIG is indicative of the so called disk-halo interaction (see Dettmar 2004, for a recent

* Based on observations obtained with XMM-Newton, an ESA science mission with instruments and contributions directly funded by ESA member states and NASA. review). Therefore, we concluded that multi-phase gaseous halos are created by star formation (SF) related processes in the disk plane. Our results presented in Paper I did not yield convincing evidence that galaxy halos form by infalling gas from an external reservoir, as considered, e.g., by Benson et al. (2000), Toft et al. (2002), or Pedersen et al. (2005).

Apart from these phenomenological analogies, there also should exist strong statistical correlations between total (disk+halo) soft X-ray, $\mathrm{H} \alpha$ (DIG), and radio continuum luminosities. If this hypothesis is correct, these luminosities should also strongly correlate with other star formation indicators, such as the FIR, $B$-band, and UV luminosity (e.g., Condon 1992; Read \& Ponman 2001), but also with direct tracers of massive star formation, such as star formation rates (SFRs, see Kennicutt 1998), supernova ( $\mathrm{SN}$ ) energy input rates normalized to the area of active star formation $\dot{E}_{\mathrm{A}}^{\text {tot }}$ (e.g., Dahlem et al. 1995; Rossa \& Dettmar 2003, simply called energy input rates, hereafter), and $L_{\mathrm{FIR}} / D_{25}^{2}$ ratios (Lehnert \& Heckman 1996). 
In the following, we investigate the interrelations among star-formation-related key parameters and luminosities to establish significant (statistical) correlations of the up to now purely morphological relations.

Integrated luminosities are used to test whether the diffuse emission from the disk and halo is coupled to SF, whereas the pure extraplanar soft X-ray emission is put into relation to the above-mentioned parameters to show that multi-phase halos are indeed tightly correlated with SF processes in the disk.

Finally, we discuss the question of the existence of an energy threshold provided by stellar feedback processes, which needs to be exceeded in order to create multi-phase halos. From the above-mentioned correlations, first empirical estimates of the suspected energy threshold are derived.

\section{Statistical analysis of key parameters}

\subsection{Key parameters and sample selection}

It is straightforward to include additional quantities that trace massive stars in the disk and the energy they provide for the interstellar medium (ISM) and to search for trends among all these parameters. Such supplemental key quantities considered here are the $B$-band and UV luminosites, as well as SFRs (or the FIR SFR per unit area, expressed by $L_{\mathrm{FIR}} / D_{25}^{2}$ ) and energy input rates by $\mathrm{SNe}$, represented by $\dot{E}_{\mathrm{A}}^{\text {tot }}$.

We also include variables that are not directly coupled to SF, but trace different neutral phases of the ISM, such as the dust mass and the H I mass of a galaxy.

Moreover, the extent of the $\mathrm{SN}$-driven gas and therefore the size of the multi-phase halo also depends on the gravitational potential of a galaxy (e.g., Mac Low \& Ferrara 1999). As a first order approximation of this quantity, the baryonic mass of a galaxy (stars and $\mathrm{HI}$ ) determined from the $K$-band Tully-Fisher relation (Bell \& de Jong 2001) is also included.

As was already pointed out in Paper I, our initial sample is biased against starburst galaxies, that is, towards higher SFRs or energy input rates $\dot{E}_{\mathrm{A}}^{\text {tot }}$. In order to investigate the possible correlations, non-starburst (but actively star-forming) galaxies need to be included and they have to cover the intermediate and lower energetic ends of these parameters. This obstacle was overcome by selecting edge-on $\left(i>70^{\circ}\right)$ late-type spiral galaxies from the literature for which the above-mentioned luminosities have been published and the SF-related parameters could be calculated. In order to reach the largest possible coverage of the parameter space, no constraints were imposed on the integrated luminosities, the $S 60 / S 100$ and $L_{\mathrm{FIR}} / D_{25}^{2}$ ratios, and the energy input rates $\dot{E}_{\mathrm{A}}^{\text {tot }}$.

Galaxies are considered to have multi-phase gaseous halos, if they show kpc-sized extraplanar radio continuum, DIG, and $\mathrm{X}$-ray emission that surrounds a large fraction of the disk plane. Extended X-shaped DIG structures, as visible on $\mathrm{H} \alpha$ images of NGC 1482 (Strickland et al. 2004a) or NGC 5775 (Tüllmann et al. 2006), most likely trace a limb-brightened outflow cone and are also classified as possessing a halo. Single plumes or filamentary structures are not considered to constitute a halo.

It should be kept in mind that the sample is limited in sensitivity and is not complete.

The enlarged sample now consists of 23 galaxies, nine from Tüllmann et al. (2006), seven from Strickland et al. (2004a, three of their targets are also in our sample and their results are consistent with ours), and another seven galaxies taken from the literature. The number of targets is sufficiently large to test the individual correlations by means of a statistical analysis. All physical parameters adopted throughout this study are listed for the whole sample in Table 1.

Integrated X-ray luminosities for the first nine galaxies listed in Table 1 are from Paper I and the next seven are taken from Strickland et al. (2004a), whereas the last seven stem from other literature sources (see notes to table).

\subsection{Statistical analysis}

We performed a least-squares fitting (assuming $Y=m X+b$ ) and Spearman rank-order correlation analysis to test the significance of the correlation between the investigated pairs of parameters. As these quantities, for example $L_{X}$ and $L_{\mathrm{FIR}}$, usually vary only within a certain physical range, e.g., set by the number of stars in the disk, these variables are most likely not normally distributed and their interrelation needs to be investigated by means of a rank-order correlation analysis.

The slopes $m$, their intercepts $b$ and $b^{\prime}$ (for $m=1.0$ ), the reduced $\chi^{2}$, the Spearman rank-order correlation coefficients $r_{\mathrm{s}}$, and $t_{\mathrm{s}}$, the levels of significance of $r_{\mathrm{s}}$, and the corresponding $p$-values of $r_{\mathrm{s}}$ are listed in Table 2 . In the case that both quantities are not linearly correlated (null hypothesis), $t_{\mathrm{s}}$ follows a Student's $t$-distribution with $n-2$ degrees of freedom. The null hypothesis turns out to be wrong if $\left|t_{\mathrm{s}}\right|>t_{\mathrm{n}-2,1-\alpha}$, where $\alpha$ is the level of significance, which we adopt to be 0.01 . The quantile $t_{21,0.99}$ of the $t$-distribution, as tabulated in relevant statistics textbooks (e.g., Hartung et al. 2002), is 2.518. For all $t_{\mathrm{s}}$-values that are larger, the null hypothesis needs to be discarded (with a 99\% confidence level), which means that all those pairs of parameters are strongly correlated.

Moreover, if the $p$-values listed in Table 2 for the integrated luminosities are less than the adopted $\alpha$-level, the null hypothesis needs to be discarded, too. In the present case only H I and baryonic masses do not fulfill these criteria and are therefore assumed not to correlate with soft X-ray luminosities.

The same analysis was carried out for the extraplanar soft $\mathrm{X}$-ray emission $\left(L_{\mathrm{X}, \mathrm{h}}\right)$ and the derived parameters are also listed in Table 2. All correlations and their corresponding least-square fits are shown in Fig. 3.

\section{Results and discussion}

\subsection{Correlations with integrated luminosities}

Based upon statistical analysis of the sample, strong linear correlations $\left(r_{\mathrm{s}} \geq 0.67\right)$ between star formation indicators and integrated (disk+halo) multi-frequency luminosities could be established. In addition to the generally accepted $L_{\mathrm{FIR}} / L_{1.4 \mathrm{GHz}} \mathrm{re}-$ lation (e.g., de Jong et al. 1985; Condon 1992, see also Table 2 of this work), highly significant linear dependencies between integrated soft X-ray luminosities $(0.3-2.0 \mathrm{keV})$ and integrated radio continuum $(1.4 \mathrm{GHz}), B$-band, UV, $\mathrm{H} \alpha$, and FIR luminosities have been found (see Fig. 1) ${ }^{1}$. Moreover, the integrated soft $\mathrm{X}$-ray luminosities correlate well with star formation rates and $\mathrm{SNe}$ energy input rates (Figs. 1 and 2). These correlations unambiguously confirm that the diffuse radiation of the X-ray emission and the DIG, as well as the CRs detected in the disks and halos, are indeed coupled to SF.

Although $\mathrm{H} \alpha, \mathrm{B}$, and UV-band luminosities are generally a good tracer of the SF activity, these quantities can be seriously

\footnotetext{
1 The latter two relations are not shown here, as the $\mathrm{H} \alpha$ and FIR luminosities are directly proportional to the corresponding SFRs (see Kennicutt 1998).
} 


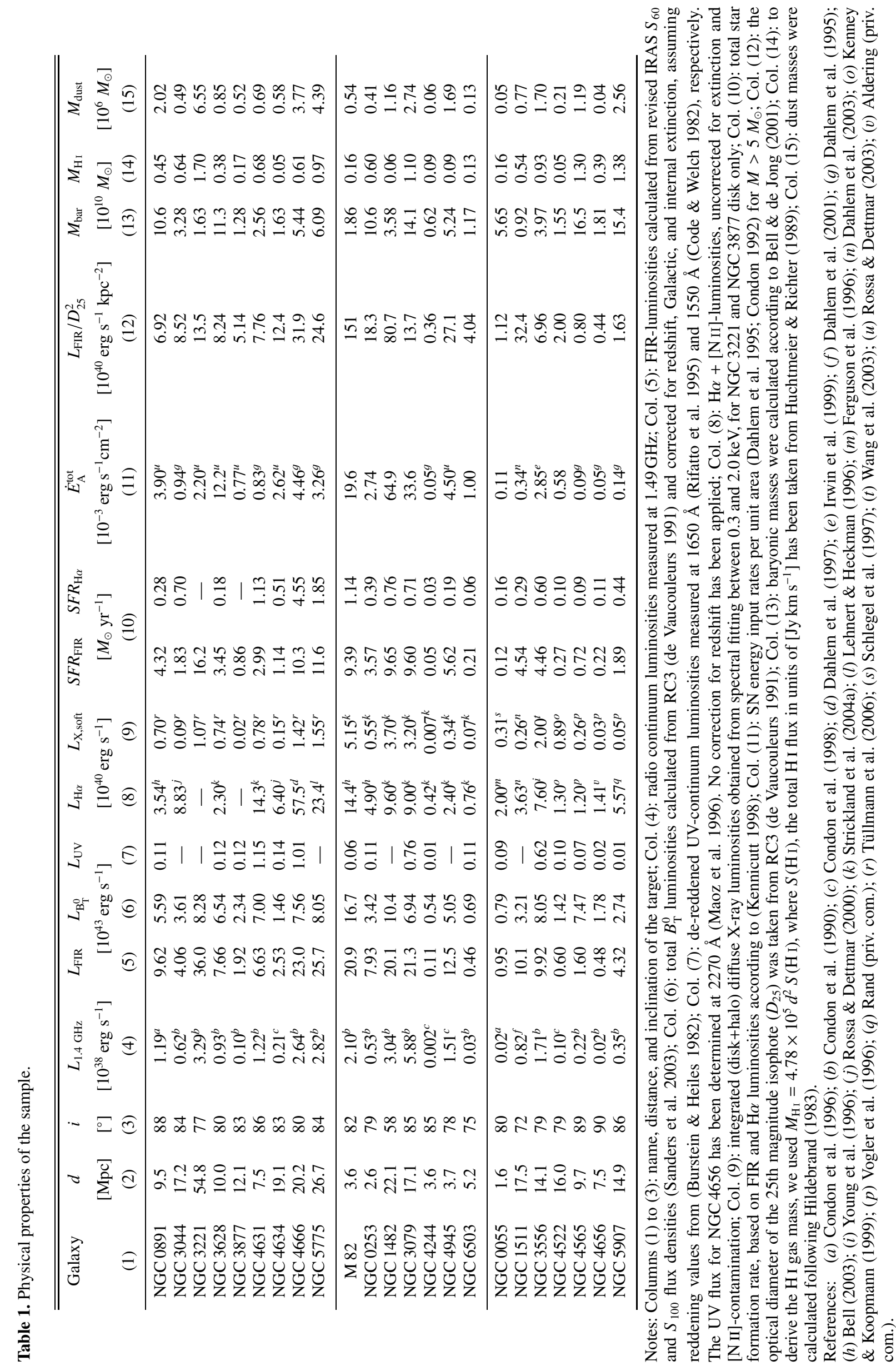


Table 2. Fit parameters and correlation coefficients. Listed are the Spearman rank-order correlation coefficient $r_{\mathrm{s}}$, its corresponding significance $t_{\mathrm{s}}$, and the $p$-value of $r_{\mathrm{s}}$, together with results derived from linear regression $(Y=m X+b)$ for the pairs of parameters plotted in Figs. 1-3. Uncertainties of the gradient $m$ and the intercept $b$ are given on a $1 \sigma$ level. $b^{\prime}$ is the intercept if a slope of $m=1$ is assumed.

\begin{tabular}{llccccccr}
\hline \hline $\log (Y)$ & $\log (X)$ & $m$ & $b$ & $b^{\prime}$ & red. $\chi^{2}$ & $r_{\mathrm{s}}$ & $t_{\mathrm{s}}$ & $p$-value \\
\hline$L_{\mathrm{FIR}}$ & $L_{1.4 \mathrm{GHz}}$ & $0.94 \pm 0.06$ & $+0.85 \pm 0.04$ & +0.86 & 0.030 & 0.97 & 17.4 & $<0.0001$ \\
$L_{\mathrm{X}}$ & $L_{1.4 \mathrm{GHz}}$ & $0.65 \pm 0.11$ & $-0.10 \pm 0.11$ & -0.01 & 0.266 & 0.74 & 4.80 & 0.0001 \\
$L_{\mathrm{X}}$ & $L_{\mathrm{FIR}}$ & $0.80 \pm 0.14$ & $-0.87 \pm 0.13$ & -0.99 & 0.243 & 0.76 & 5.10 & 0.0001 \\
$L_{\mathrm{X}}$ & $L_{\mathrm{H} \alpha}$ & $0.88 \pm 0.27$ & $-0.95 \pm 0.22$ & -1.03 & 0.370 & 0.67 & 3.93 & 0.0009 \\
$L_{\mathrm{X}}$ & $L_{\mathrm{B}}$ & $1.29 \pm 0.29$ & $-1.12 \pm 0.19$ & -0.95 & 0.295 & 0.71 & 4.39 & 0.0003 \\
$L_{\mathrm{X}}$ & $L_{\mathrm{UV}}$ & $0.87 \pm 0.22$ & $+0.28 \pm 0.24$ & +0.40 & 0.292 & 0.73 & 4.66 & 0.0002 \\
$L_{\mathrm{X}}$ & $S F R_{\mathrm{FIR}}$ & $0.79 \pm 0.15$ & $-0.58 \pm 0.11$ & -0.64 & 0.252 & 0.76 & 5.10 & 0.0001 \\
$L_{\mathrm{X}}$ & $S F R_{\mathrm{H} \alpha}$ & $0.86 \pm 0.27$ & $-0.07 \pm 0.18$ & -0.02 & 0.378 & 0.64 & 3.63 & 0.0018 \\
$L_{\mathrm{X}}$ & $L_{\mathrm{FIR}} / D_{25}^{2}$ & $0.73 \pm 0.17$ & $-1.07 \pm 0.19$ & -1.32 & 0.303 & 0.69 & 4.16 & 0.0005 \\
$L_{\mathrm{X}}$ & $\dot{E}_{\mathrm{A}}$ & $0.68 \pm 0.12$ & $-0.47 \pm 0.10$ & -0.51 & 0.230 & 0.78 & 5.43 & $<0.0001$ \\
$L_{\mathrm{X}}$ & $M_{\mathrm{dust}}$ & $0.70 \pm 0.21$ & $-0.27 \pm 0.13$ & -0.22 & 0.386 & 0.58 & 3.10 & 0.0058 \\
$L_{\mathrm{X}}$ & $M_{\mathrm{H} \mathrm{I}}$ & $0.15 \pm 0.33$ & $-0.31 \pm 0.22$ & +0.09 & 0.578 & 0.09 & 0.39 & 0.6980 \\
$L_{\mathrm{X}}$ & $M_{\mathrm{Baryon}}$ & $0.51 \pm 0.37$ & $-0.66 \pm 0.25$ & -0.93 & 0.535 & 0.29 & 1.32 & 0.2022 \\
\hline$L_{\mathrm{X}, \mathrm{h}}$ & $S F R_{\mathrm{FIR}}$ & $0.93 \pm 0.17$ & $-1.13 \pm 0.14$ & -1.11 & 0.189 & 0.72 & 3.42 & 0.0057 \\
$L_{\mathrm{X}, \mathrm{h}}$ & $S F R_{\mathrm{H} \alpha}$ & $1.13 \pm 0.22$ & $-0.31 \pm 0.15$ & -0.35 & 0.203 & 0.71 & 3.26 & 0.0075 \\
$L_{\mathrm{X}, \mathrm{h}}$ & $\dot{E}_{\mathrm{A}}$ & $0.77 \pm 0.21$ & $-1.12 \pm 0.19$ & -1.14 & 0.308 & 0.69 & 2.96 & 0.0144 \\
$L_{\mathrm{X}, \mathrm{h}}$ & $L_{\mathrm{FIR}} / D_{25}^{2}$ & $0.70 \pm 0.32$ & $-1.60 \pm 0.45$ & -1.89 & 0.449 & 0.61 & 2.45 & 0.0341 \\
\hline & & & & & & & & \\
\hline
\end{tabular}

affected by absorption internal to the galaxy. The typical scatter of about 1 dex visible in our correlations might be indicative of this effect. However, absorption appears to be unlikely to lower the luminosities in such a way that the correlations are seriously weakened or even destroyed.

The strongly discrepant data point visible in the $L_{\mathrm{X}} / L_{\mathrm{UV}}$-diagram represents measurements for one of the most extreme starburst galaxies, M 82. For M 82 to follow our correlation, a much higher (lower) UV (X-ray) luminosity of about $1 \mathrm{dex}$ is required. However, independent observations carried out in the UV (Code \& Welch 1982; Rifatto et al. 1995; Hoopes et al. 2005) and at X-ray energies (Fabbiano et al. 1992; Strickland et al. 2004a) yield very consistent results which imply that this "deviation" is an intrinsic feature of this outstanding galaxy.

More than $60 \%$ of the UV emission of M 82 measured at about $1530 \AA$ is extraplanar in origin (Hoopes et al. 2005). These authors claim that extraplanar star formation or a combination of photoionization and shock ionization either appear unlikely or cannot account for the halo emission in the UV. Instead, stellar continuum radiation from the disk plane scattered at dust particles in the halo shall be the main mechanism causing the UV emission. Given that M 82 is a low mass spiral galaxy $\left(M_{\text {bar }}=1.86 \times 10^{10} M_{\odot}\right.$, Table 1$)$, dust is assumed to be driven relatively easily by the superwind to high extraplanar distances. This independently supports the "scattering"-hypothesis and could also explain the lacking UV emission, provided the $\left(L_{\mathrm{X}}, L_{\mathrm{UV}}\right)$-relation is valid.

Alternatively, the high X-ray and low UV luminosity of M 82 can be explained by gas within the starburst region, which is thermalized beyond the UV excitation limit. In such an extreme case, in which the diffuse soft X-ray emission dominates the energy output, we expect a deviation from the linear correlation curve during phases of strong starburst activity.

Apparently only a weak correlation between the dust mass of a galaxy and the corresponding soft X-ray luminosity exists. Dust, as a byproduct of SF, is correlated with the FIR luminosity and should therefore also correlate (at least weakly) with other SF indicators, such as X-ray luminosities.
The non-correlation between $L_{\mathrm{X}}$ and $M_{\mathrm{HI}}$ is also not surprising as the neutral gas component of the ISM is not directly involved in SF.

Surprising only at a first glance, a correlation between galaxy potential and X-ray luminosities is not found. As the baryonic mass (Bell \& de Jong 2001) is calculated from rotational velocities of the stars and the H I-gas in a galaxy, this parameter traces the dynamics of the system and is therefore insensitive to SF. Nevertheless, this finding bears two important implications. Firstly, the dynamics of the gas is apparently not related to the diffuse emission produced in SF events. Secondly, for a more accurate estimate of the baryonic mass, a better mass-related approximation needs to be found. This relation should also include dark matter halos to study the effects this additional component might have on the established relations. If there is still no correlation with baryonic mass, then the targets of our sample do not follow the $L_{\mathrm{X}} \sim v_{\text {rot }}^{5}$ relation derived from the infall simulations by Toft et al. (2002).

It should be pointed out that $M_{\text {bar }}$ represents the gravitational potential of a galaxy and should therefore be sensitive to the overall extent of the multi-phase halo. However, it is not sensitive to the existence/non-existence of such halos. In this regard, the SFR and the energy input rate by SNe into the ISM turn out to be the most important parameters.

\subsection{Correlations with extraplanar luminosities}

In order to establish that multi-phase halos are indeed a product of SF, the pure extraplanar emission in the radio continuum, in $\mathrm{H} \alpha$, and at soft X-ray energies also needs to correlate with SFRs and the energy input rate by $\mathrm{SNe}$.

At present, this analysis can best be done in the X-ray regime. Other wavelengths, such as $\mathrm{H} \alpha$ and the radio continuum, are not usable, because the integral disk and halo luminosities do not allow us to reliably estimate the fraction of the luminosity originating in the halo. For the DIG, this fraction can vary from $12 \%$ to $60 \%$ (Rossa \& Dettmar 2000) and would therefore lead to unacceptable uncertainties. 

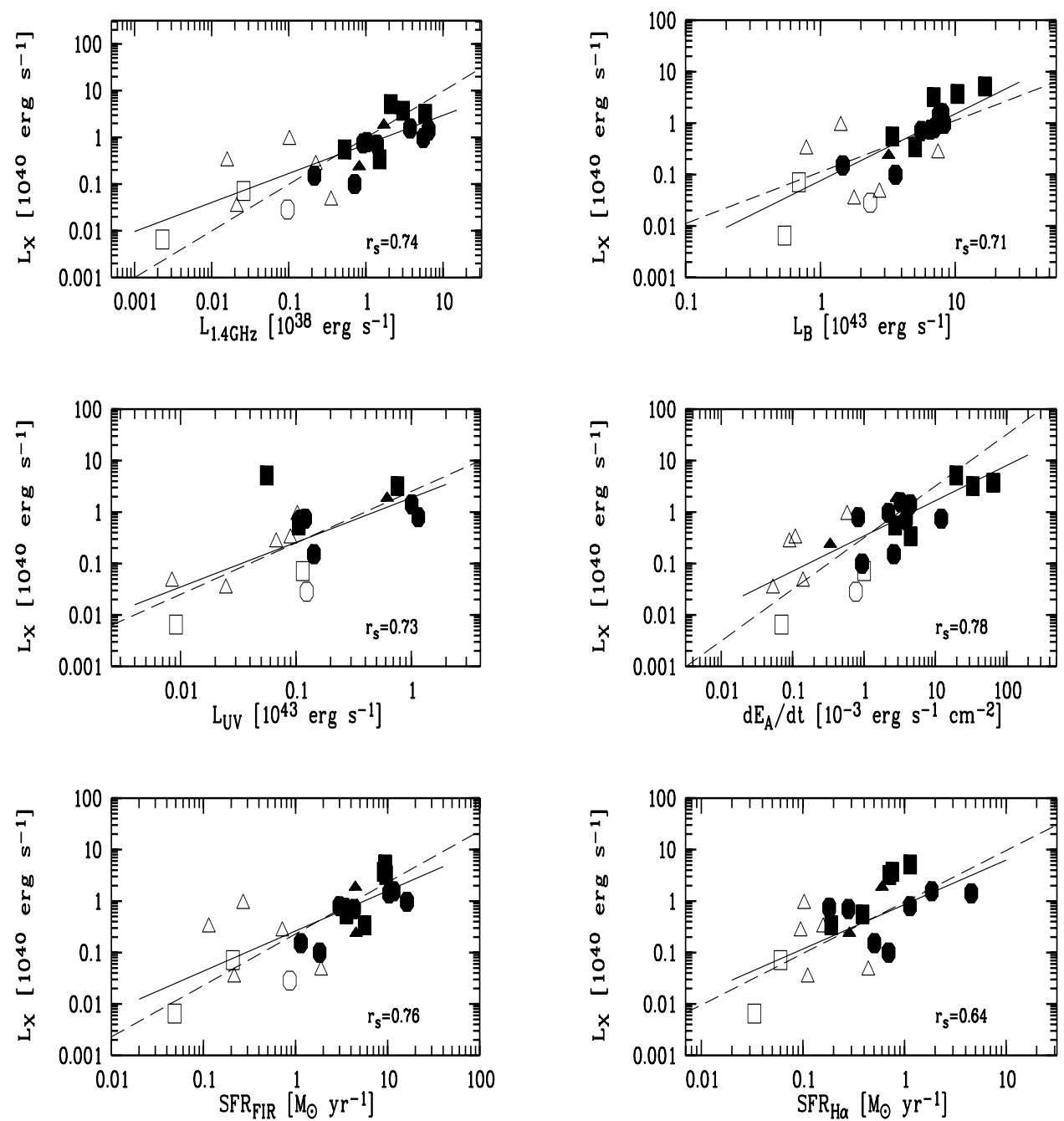

Fig. 1. Diagnostic diagrams confirming the strong correlations between integrated radio continuum, $B$-band, $\mathrm{UV}$, and soft $\mathrm{X}$-ray $(0.3-2.0 \mathrm{keV})$ luminosities. X-ray luminosities also correlate well with the energy input rate by SNe $\left(\dot{E}_{\mathrm{A}}\right)$ and with FIR and $\mathrm{H} \alpha$ SFRs. Circles address our sample, squares represent data from Strickland et al. (2004b), and triangles denote data collected from the literature (cf. Table 1). Filled (open) symbols refer to galaxies with (un)detected multi-phase gaseous halos. Solid lines are best fits from linear regression, while dashed lines indicate the trend expected for a relationship of unit slope. $r_{\mathrm{s}}$ is the Spearman rank-order correlation coefficient.

From the original sample, 13 galaxies are suitable for the "halo"-sample (those from Strickland et al. 2004a; and Tüllmann et al. 2006). The remaining galaxies either do not show extraplanar X-ray emission or the published data did not allow a disentangling of disk and halo luminosities.

It should be pointed out that inclination issues, regarding the reliability of detecting multi-phase halos, are negligible. Extraplanar gas was detected in cases of inclination angles $\leq 80^{\circ}$ (see, e.g., NGC 1482, Strickland et al. 2004a), or the inclinationcorrected extent of the "extraplanar" gas was indeed not large enough to be seen above the disk plane (see, e.g., NGC 3877, Tüllmann et al. 2006). Most importantly, $L_{\mathrm{X}}$ and $L_{\mathrm{X}, \mathrm{h}}$ are not linearly correlated with the inclination angle $i$. This is proved by low Spearman rank-order correlation coefficients of $r_{\mathrm{s}}=-0.26$ and $r_{\mathrm{s}}=0.23$, respectively, again on a $99 \%$ confidence level.

In Fig. 3 we plot the soft $(0.3-2.0 \mathrm{keV})$ halo luminosity $\left(L_{\mathrm{X}, \mathrm{h}}\right)$ as a function of the different SF indicators (which are $S F R_{\mathrm{FIR}}, S F R_{\mathrm{H} \alpha}, L_{\mathrm{FIR}} / D_{25}^{2}$, and $\left.\dot{E}_{\mathrm{A}}\right)$. All pairs of parameters show strong correlations $\left(r_{\mathrm{s}} \geq 0.67\right)$ on a 99\% confidence level, except $L_{\mathrm{X}, \mathrm{h}}$ vs. $L_{\mathrm{FIR}} / D_{25}^{2}\left(r_{\mathrm{s}}=0.61\right)$. A possible explanation could be that $L_{\mathrm{FIR}} / D_{25}^{2}$, a measure of the SF rate intensity per unit disk area, underestimates the real SF intensity. In disk galaxies,
SF usually occurs within the $\mathrm{H} \alpha$-disk and is not as widespread as the distribution of the old stellar population (measured by $D_{25}$ ) implies.

It turns out that the FIR and the $\mathrm{H} \alpha$ SFRs are the most important parameters that decide on the formation of X-ray halos. Among these two quantities, $S F R_{\text {FIR }}$ seems to be the more reliable parameter, because it is very little affected by dust absorption and is dominated by disk emission. On the other hand, the $\mathrm{H} \alpha$ emisson, and therefore $S F R_{\mathrm{H} \alpha}$, is a more sensitive measure of how much gas is converted into massive stars. However, this quantity is significantly affected by extinction, and in some cases, most likely dominated by extraplanar emission.

Given the strong correlations between integrated X-ray, $\mathrm{H} \alpha$, and radio continuum luminosities, as well as between halo X-ray luminosities and SFRs or $\dot{E}_{\mathrm{A}}$, it is plausible to adopt similar relations for the extraplanar DIG and the CR halos. Therefore, we expect the SFR and the energy input rate by SNe to be the main driving-agents for multi-phase galaxy halos. Our statistical results are also fully consistent with the morphological and spatial coincidences presented in Paper I. For consistency reasons, it is certainly worthwhile to re-examine existing radio and DIG data to allow for a clear separation of disk and halo luminosities. 
R. Tüllmann et al.: The multi-phase gaseous halos of star-forming late-type galaxies. II.
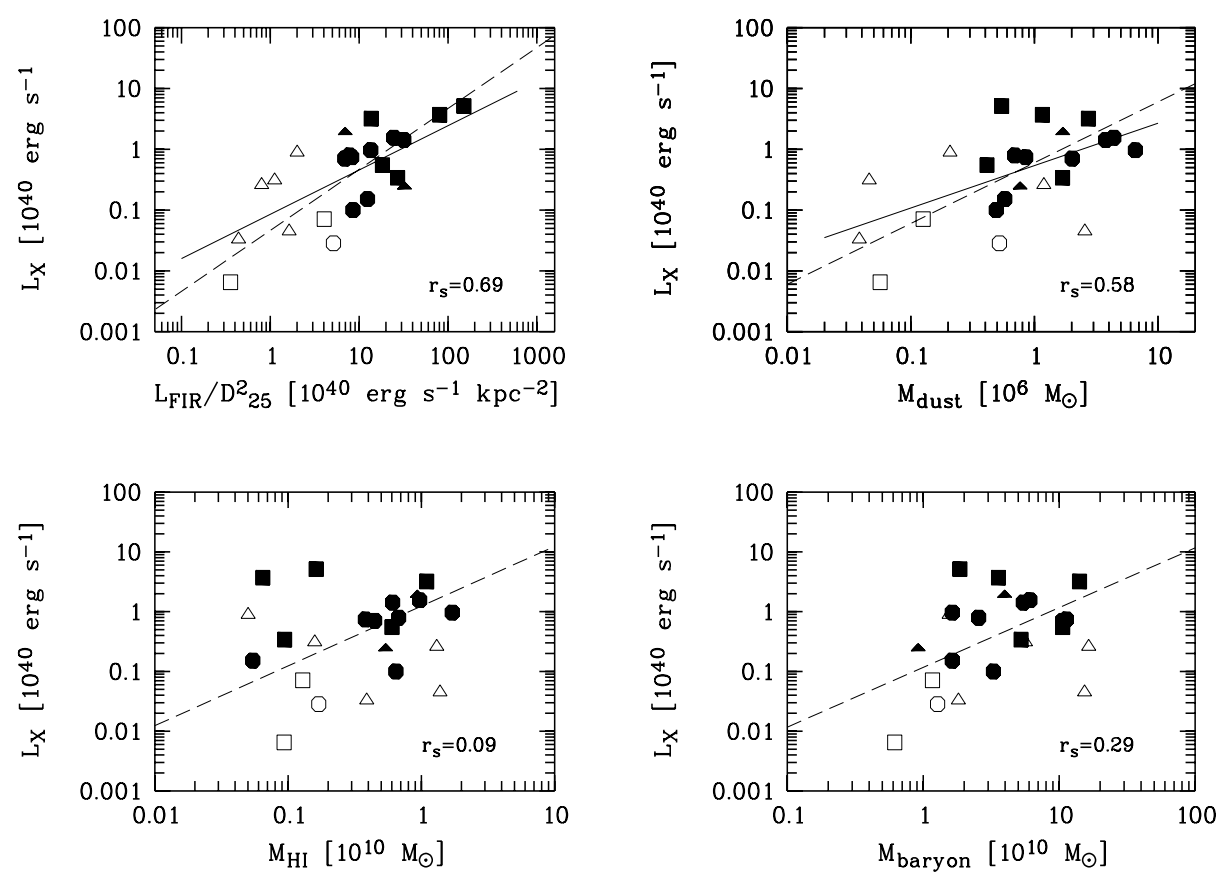

Fig. 2. Functional dependence between soft X-ray luminosities and $L_{\mathrm{FIR}} / D_{25}^{2}$ (upper left), the dust mass of a galaxy (upper right), its $\mathrm{H}$ I mass (lower left), and the baryonic mass (lower right).
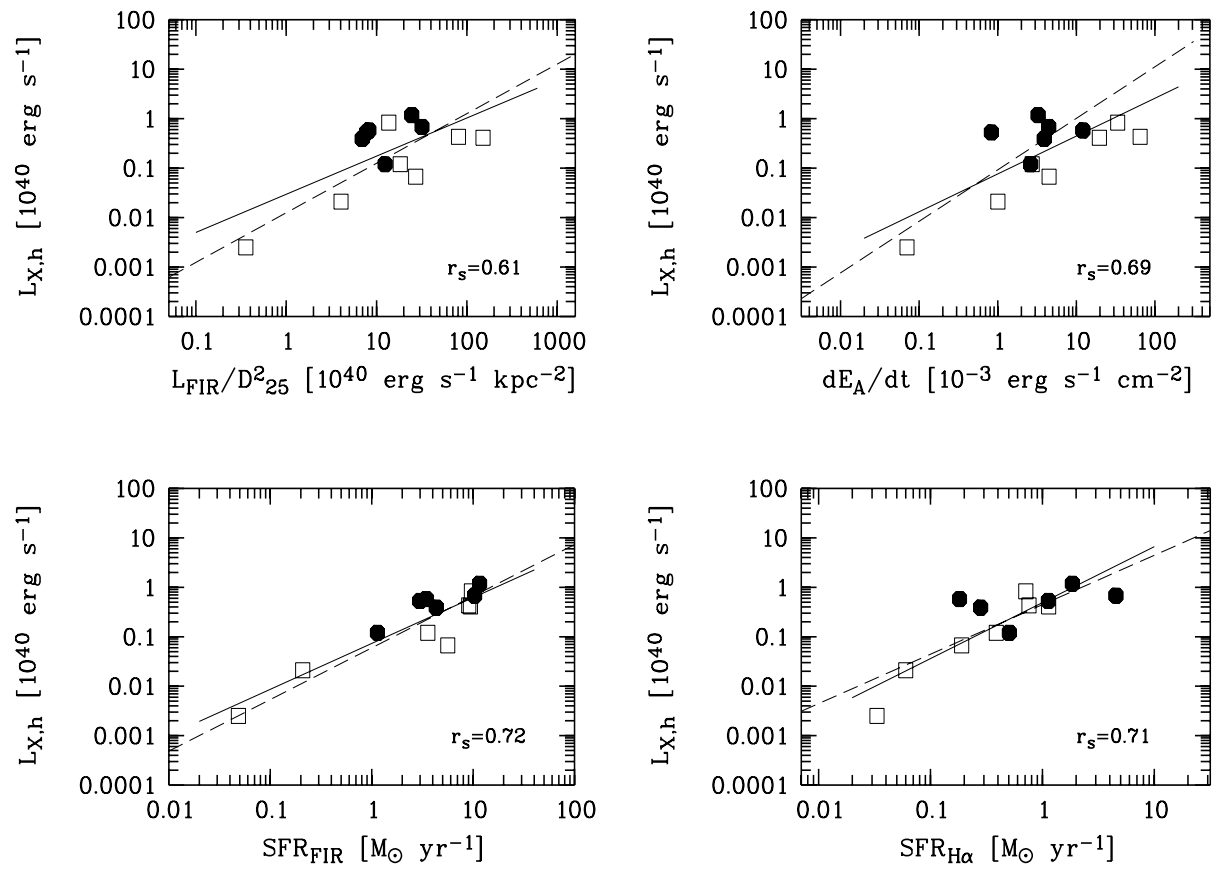

Fig. 3. Functional dependence between $L_{\mathrm{X}, \mathrm{h}}$, the soft $(0.3-2.0 \mathrm{keV}) \mathrm{X}$-ray luminosity of the halo, and $L_{\mathrm{FIR}} / D_{25}^{2}$ (upper left), $\dot{E}_{\mathrm{A}}$ (upper right), $S F R_{\mathrm{FIR}}$ (lower left), and $S F R_{\mathrm{H} \alpha}$ (lower right). Solid symbols represent our sample, open symbols denote data taken from Strickland et al. (2004a). Solid and dashed lines have the same meaning as described in Fig. 1.

\subsection{Constraining the star formation threshold}

In order to achieve a more comprehensive picture of the evolution of galaxy halos in different wave bands, we need to answer the question of the existence of a threshold SFR (or equivalently the $\mathrm{SN}$ energy input rate $\dot{E}_{\mathrm{A}}$ ) required to create multi-phase gaseous halos. The existence of such a threshold can be motivated as follows: It is well-known that hot ionized gas is driven off-plane via superbubbles produced by SNe. From a theoretical study of breakout conditions of such superbubbles
(Mac Low \& Mc Cray 1988), we know that the mechanical luminosity of $\mathrm{SNe}$ is a key quantity that determines whether a superbubble will breakout or collapse. In other words, the overpressured X-ray emitting gas will leave the disk and form a halo if the energy provided by $\mathrm{SNe}$ is above a certain limit.

On the other hand, it can be argued that channels, through which the hot gas reaches the halo, were already blown into the ISM during previous periods of intense star formation. Hence, a substantially lower SFR is needed to elevate the gas into the 
halo, making it even more difficult to establish a threshold by means of observations.

From Fig. 1, the existence of a critical SFR (or energy input rate) is hard to constrain, as the lower energy end of our correlations is statistically not well covered. It appears, however, that galaxies without halos (open symbols) also follow a linear relation and that the region dividing galaxies with halos from those without is relatively narrow. For multi-phase halos to evolve, the data imply that a critical threshold of $S F R_{\mathrm{FIR}} \geq 1.0 M_{\odot} / \mathrm{yr}$, $S F R_{\mathrm{H} \alpha} \geq 0.1 M_{\odot} / \mathrm{yr}$, or $\dot{E}_{\mathrm{A}} \geq 1 \times 10^{-3} \mathrm{erg} \mathrm{s}^{-1} \mathrm{~cm}^{-2}$ needs to be exceeded. Interestingly, DIG seems to coexist with other gas components for $\mathrm{H} \alpha$ energy fluxes larger than $(3.2 \pm 0.5) \times$ $10^{40} \mathrm{erg} \mathrm{s}^{-1} \mathrm{kpc}^{-2}$ (Rossa \& Dettmar 2003).

Unfortunately, with the present data a detection threshold, introducing systematic uncertainties by the limited sensitivity of the instrument, cannot a priori be ruled out. In order to distinguish between a significant physical threshold and a sensitivity limit, as well as to reach a better understanding on the formation of gaseous galaxy halos, deep observations of galaxies with only little or no halo emission are required. Therefore, future studies should aim at increasing the sample to test the low energy end of our correlations and to investigate the above relations for galaxies of different Hubble-types, such as Sa and Irr, as the different SF histories of these galaxies might lead to different relations and thresholds.

\section{Summary and conclusions}

With a sample of 23 actively star-forming galaxies studied in the X-ray regime and by implementing additional wave bands (H $\alpha$ and UV), we found remarkably strong linear correlations between integrated $1.4 \mathrm{GHz}$ radio continuum, FIR, $\mathrm{H} \alpha, B$-band, $\mathrm{UV}$, and soft X-ray luminosities. Strong correlations also exist if soft X-ray luminosities are plotted against SFRs, $L_{\mathrm{FIR}} / D_{25}^{2}$, or the energy input rate by $\mathrm{SNe}$ per unit area, expressed by $\dot{E}_{\mathrm{A}}$. Integrated X-ray luminosities neither correlate with the H I nor with the baryonic mass of a galaxy.

Strong correlations are also found if the diffuse soft X-ray luminosity of the halo is plotted against the FIR and $\mathrm{H} \alpha$ SFR or the SN energy input rate $\dot{E}_{\mathrm{A}}$. These quantities are considerd to be the most important parameters for the creation of multi-phase halos.

If a critical energy threshold exists and an instrumental detection bias is negligible, the present data suggest that a threshold of $S F R_{\mathrm{FIR}} \geq 1.0 M_{\odot} / \mathrm{yr}, S F R_{\mathrm{H} \alpha} \geq 0.1 M_{\odot} / \mathrm{yr}$, or $\dot{E}_{\mathrm{A}} \geq$ $1 \times 10^{-3} \mathrm{erg} \mathrm{s}^{-1} \mathrm{~cm}^{-2}$ needs to be exceeded to create multi-phase galaxy halos.

Our results are in agreement with previous findings of a morphological and spatial coincidence of gaseous multi-phase halos (see Paper I). They clearly imply that multi-phase halos are the consequence of stellar feedback processes in the disk plane (e.g., de Avillez \& Breitschwerdt 2004, 2005), but conflict with the concept of halos being due to infalling gas from the intergalactic medium (e.g., Benson et al. 2000; Toft et al. 2002; Pedersen et al. 2005).
Acknowledgements. R.T. acknowledges financial support by Deutsches Zentrum für Luft- und Raumfahrt (DLR) through grant 50 OR 0102. We appreciate the comments made by the anonymous referee. This work has made use of the SIMBAD database and of HyperLeda (http://leda. univ-lyon1. fr/).

\section{References}

Bell, E. F. 2003, ApJ, 586, 794

Bell, E. F., \& de Jong, R. S. 2001, ApJ, 550, 212

Benson, A. J., Bower, R. G., Frenk, C. S., \& White, S. D. M. 2000, MNRAS, 314,557

Burstein, D., \& Heiles, C. 1982, AJ, 87, 1165

Code, A., \& Welch, G. 1982, ApJ, 256, 1

Condon, J. J. 1992, ARA\&A, 30, 575

Condon, J. J., Helou, G., Sanders, D. B., \& Soifer, B. T. 1990, ApJS, 73, 359

Condon, J. J., Helou, G., Sanders, D. B., \& Soifer, B. T. 1996, ApJS, 103, 81

Condon, J. J., Cotton, W. D., Greisen, E. W., et al. 1998, AJ, 115, 1693

Dahlem, M., Lisenfeld, U., \& Golla, G. 1995, ApJ, 444, 119

Dahlem, M., Petr, M., Lehnert, M. D., Heckman, T. M., \& Ehle, M. 1997, A\&A, 320, 731

Dahlem, M., Lazendic, J. S., Haynes, R. F., Ehle, M., \& Lisenfeld, U. 2001, A\&A, 374, 42

Dahlem, M., Ehle, M., Jansen, F., et al. 2003, A\&A, 403, 547

de Avillez, M. A., \& Breitschwerdt, D. 2004, A\&A, 425, 899

de Avillez, M. A., \& Breitschwerdt, D. 2005, A\&A, 436, 585

de Jong, T., Klein, U., Wielebinski, R., \& Wunderlich, E. 1985, A\&A, 147, L6

Dettmar, R.-J. 2004, Ap\&SS, 289, 349

de Vaucouleurs, G., et al. 1991, Third Reference Catalogue of Bright Galaxies (RC3) (Springer Verlag)

Fabbiano, G., Kim, D.-W., \& Trinchieri, G. 1992, ApJS, 80, 531

Ferguson, A. M. N., Wyse, R. F. G., Gallagher, J. S. III, \& Hunter, D. A. 1996, AJ, 112,2567

Hartung, J., Elpelt, B., \& Klösener, K.-H. 2002, Statistik, Lehr- und Handbuch der angewandten Statistik, 13th ed., Oldenbourg Verlag

Hildebrand, R. H. 1983, QJRAS, 24, 267

Hoopes, C. G., Heckman, T. M., Strickland, D. K., et al. 2005, ApJ, 619, L99

Huchtmeier, W. K., \& Richter, O.-G. 1989, A General Catalog of H I observations of Galaxies (Springer Verlag)

Irwin, J. A., English, J., \& Sorathia, B. 1999, AJ, 117, 2102

Kenney, J. D. P., \& Koopmann, R. A. 1999, AJ, 117, 181

Kennicutt, R. C., Jr. 1998, ARA\&A, 36, 189

Lehnert, M. D., \& Heckman, T. M. 1996, ApJ, 472, 546

Mac Low, M.-M., \& Mc Cray, R. 1988, ApJ, 324, 776

Mac Low, M.-M., \& Ferrara, A. 1999, ApJ, 513, 142

Maoz, D., Filippenko, A. V., Ho, L. C., et al. 1996, ApJS, 107, 215

Pedersen, K., Rasmussen, J., Sommer-Larsen, J., et al., 2005, New Astron., 11, 465

Read, A. M., \& Ponman, T. J. 2001, MNRAS 328, 127

Rifatto, A., Longo, G., \& Capaccioli, M. 1995, A\&AS, 114, 527

Rossa, J., \& Dettmar, R.-J. 2000, A\&A, 359, 433

Rossa, J., \& Dettmar, R.-J. 2003, A\&A, 406, 493

Sanders, D. B., Mazzarella, J. M., Kim, D.-C., Surace, J. A., \& Soifer, B. T. 2003, AJ, 126, 1607

Schlegel, D. J., Barrett, P., \& Singh, K. P. 1997, AJ, 113, 1296

Strickland, D. K., Heckman, T. M., Colbert, E. J. M., Hoopes, C. G., \& Weaver, K. A. 2004a, ApJS, 151, 193

Strickland, D. K., Heckman, T. M., Colbert, E. J. M., Hoopes, C. G., \& Weaver, K. A. 2004b, ApJ, 606, 829

Toft, S., Rasmussen, J., Sommer-Larsen, J., \& Pedersen, K. 2002, MNRAS, 335, 799

Tüllmann, R., Pietsch, W., Rossa, J., Breitschwerdt, D., \& Dettmar, R.-J. 2006, A\&A, 448, 43 (Paper I)

Vogler, A., Pietsch, W., \& Kahabka, P. 1996, A\&A, 305, 74

Wang, Q. D., Chaves, T., \& Irwin, J. A. 2003, ApJ, 598, 969

Young, J. S., Allen, L., Kenney, J. D. P., Lesser, A., \& Rownd, B. 1996, AJ, 112, 1903 